\title{
Esponjas (Demospongiae, Halichondrida) da costa do Maranhão, Brasil
}

\author{
Beatriz Mothes ${ }^{1,3}$; Maurício Alves de Campos $^{1,3}$; Cléa Beatriz Lerner ${ }^{1} \&$ \\ Maria Marlúcia Ferreira-Correia ${ }^{2}$ \\ 1. Museu de Ciências Naturais, Fundação Zoobotânica do Rio Grande do Sul, Rua Dr. Salvador França, 1427, 90690-000, Porto
Alegre, RS, Brasil. (bmothes @ fzb.rs.gov.br)
2. Laboratório de Hidrobiologia, Universidade Federal do Maranhão, Av. dos Portugueses, s/n, Campus Universitário do Bacanga,
$65080-040$, São Luís, MA, Brasil.
3. Bolsista CNPq.
}

\begin{abstract}
Sponges (Demospongiae, Halichondrida) from Maranhão coast, Brazil. Three species of Halichondrida, Dragmacidon reticulatus (Ridley \& Dendy, 1886) (Axinellidae), Myrmekioderma rea (Laubenfels, 1934) (Desmoxyidae) and Topsentia ophiraphidites (Laubenfels, 1934) (Halichondriidae), collected from 30 to $184 \mathrm{~m}$ depths, were recorded for the first time from State of Maranhão, north-northeast coast of Brazilian shelf.
\end{abstract}

KEYWORDS. Porifera, diversity, distribution, West Atlantic.

\section{INTRODUÇÃO}

A diversidade de poríferos na costa brasileira ainda é pouco conhecida, principalmente ao norte e ao sul da desembocadura do rio Amazonas, entre o Amapá e o Maranhão; os registros dessa fauna encontram-se em Collette \& Rützler (1977), que listaram 34 táxons, dos quais apenas 18 foram identificados em nível específico; Coelho \& Mello-Leitão (1978), Hajdu \& DesqueyrouXFAÚNDEZ (1994), MotHEs et al. (1999) e MothEs et al. (2000) registraram somente uma espécie. Objetiva-se ampliar o conhecimento da diversidade de esponjas da costa do Maranhão.

\section{MATERIAL E MÉTODOS}

As amostras são provenientes de cruzeiros oceanográficos realizados pelo Programa de Avaliação do Potencial de Recursos Vivos da Zona Econômica Exclusiva (REVIZEE), entre outubro e dezembro de 1997 (REVIZEE-Norte II), junho de 1999 (REVIZEE-Norte III) e julho de 2001 (REVIZEE-Norte IV). Foram coletadas ao largo da costa do Estado do Maranhão pelo N/Oc. Antares, por meio de draga retangular e coletor VanVeen, posteriormente fixadas em formol. Amostras encontramse conservadas em álcool $90^{\circ}$ e depositadas na coleção de Poríferos Marinhos do Museu de Ciências Naturais, Fundação Zoobotânica do Rio Grande do Sul, Porto Alegre.

A metodologia utilizada para a preparação de lâminas de dissociação espicular segue Mothes-DEMoraes (1978). Para a observação da estrutura esqueletal foram montadas lâminas de cortes, retirando-se um fragmento perpendicular à superfície da esponja, o qual permaneceu 48 horas em xilol para diafanização. A seguir, incluiu-se o fragmento em parafina líquida purificada em estufa com temperatura em torno de $60^{\circ} \mathrm{C}$. Os blocos foram cortados de modo mais fino possível, com o auxílio de um bisturi, colocados sobre uma lâmina de vidro e imersos em xilol para desparafinização. Após a completa dissolução da parafina, os cortes foram cobertos com resina especial para microscopia e lamínula. Com auxílio de pinça ou bisturi, retirou-se uma fina película paralela à superfície, colocando-a sobre uma lâmina e pingando algumas gotas de xilol para clarificação, para a observação do arranjo espicular da superfície da esponja. Após a completa secagem, o corte foi coberto com resina especial para microscopia e lamínula.

Microfotografias do conjunto espicular foram realizadas com microscópio eletrônico de varredura (SILVA \& Mothes, 1996) e, para a arquitetura esqueletal, utilizouse microscópio óptico com câmera digital. Mensurações espiculares referem-se a mínima, média, máxima, expressas em $\mu \mathrm{m}$, largura após a barra $(/) ; \mathrm{N}=50$, exceto quando indicado.

As abreviaturas utilizadas correspondem: INV, Instituto de Investigaciones Marinas de Punta de Betín, Universidad Nacional de Colombia, Santa Marta; MCN, Museu de Ciências Naturais, Fundação Zoobotânica do Rio Grande do Sul, Porto Alegre; ZMA, Zoölogisch Museum Amsterdam, Amsterdam.

Axinellidae

\section{Dragmacidon reticulatus (Ridley \& Dendy, 1886)}

(Figs. 1-6)

Axinella reticulata RidLey \& DeNDy, 1886:481 (localidade-tipo: Bahia, Brasil); 1887:184,185, est. 37, figs. 4 a-b; Hechtel, 1976:253.

Axinella lunaecharta; Hechtel, 1976:252 [non A. lunaecharta RidLEy \& DENDY, 1886].

Pseudaxinella lunaecharta; Collette \& RÜtzleR, 1977:309 [examinado]; Solé-CAva et al., 1981:131, fig. 9; MotHES-DEMoraes, 1987:131, 132, figs. 3-7 [examinado]; Muricy et al., 1991:1187 [non P. lunaecharta RidLey \& Dendy, 1886].

Pseudaxinella reticulata; LERNER, 1996:110, 111 [examinado]; LeHnert \& SOESt, 1998:85; Moraes et al., 2003:17.

Dragmacidon reticulatus; Alvarez \& Hooper, 2002:734,735, fig. 9a.

Sinonímia adicional, Alvarez et al. (1998). 
Material examinado. BRASIL, Maranhão: 01 33 '81'S$43^{\circ} 15^{\prime} 87^{\prime \prime} \mathrm{W}, 80 \mathrm{~m}, 02 . X I I .1997$ (MCN 3804); 0045'25' S44³4'39'”, 43 m, 12.VI.1999 (MCN 4775).

Esponja maciça, irregular (fig. 1). Dimensões do maior espécime (MCN 3804), em cm: comprimento 5,0, largura 5,0, altura 4,0. Superfície lisa ao tato, discretamente conulosa em algumas regiões da porção inferior da esponja. Ectossoma destruído no restante, coanossoma permanece exposto. Interior com estruturas coniformes híspidas, interligadas por membranas contínuas; ósculos situados entre estruturas coniformes, alguns obstruídos por fina membrana translúcida; ósculos $0,1-0,3 \mathrm{~cm}$ em diâmetro. Material preservado de consistência pouco compressível; coloração bege-clara.

Esqueleto (fig. 2) plumoreticulado, com feixes de escleras ascendentes $(60-700 \mu \mathrm{m})$, perpendiculares à superfície, feixes algumas vezes unidos por uma ou mais escleras dispostas em ângulos variados.

Óxeas (figs. 3, 4) com extremidades gradualmente aceradas e discreta curvatura na porção mediana do eixo. Estilos (figs. 5,6) discretamente curvados na porção basal. Dimensões (tab. I).
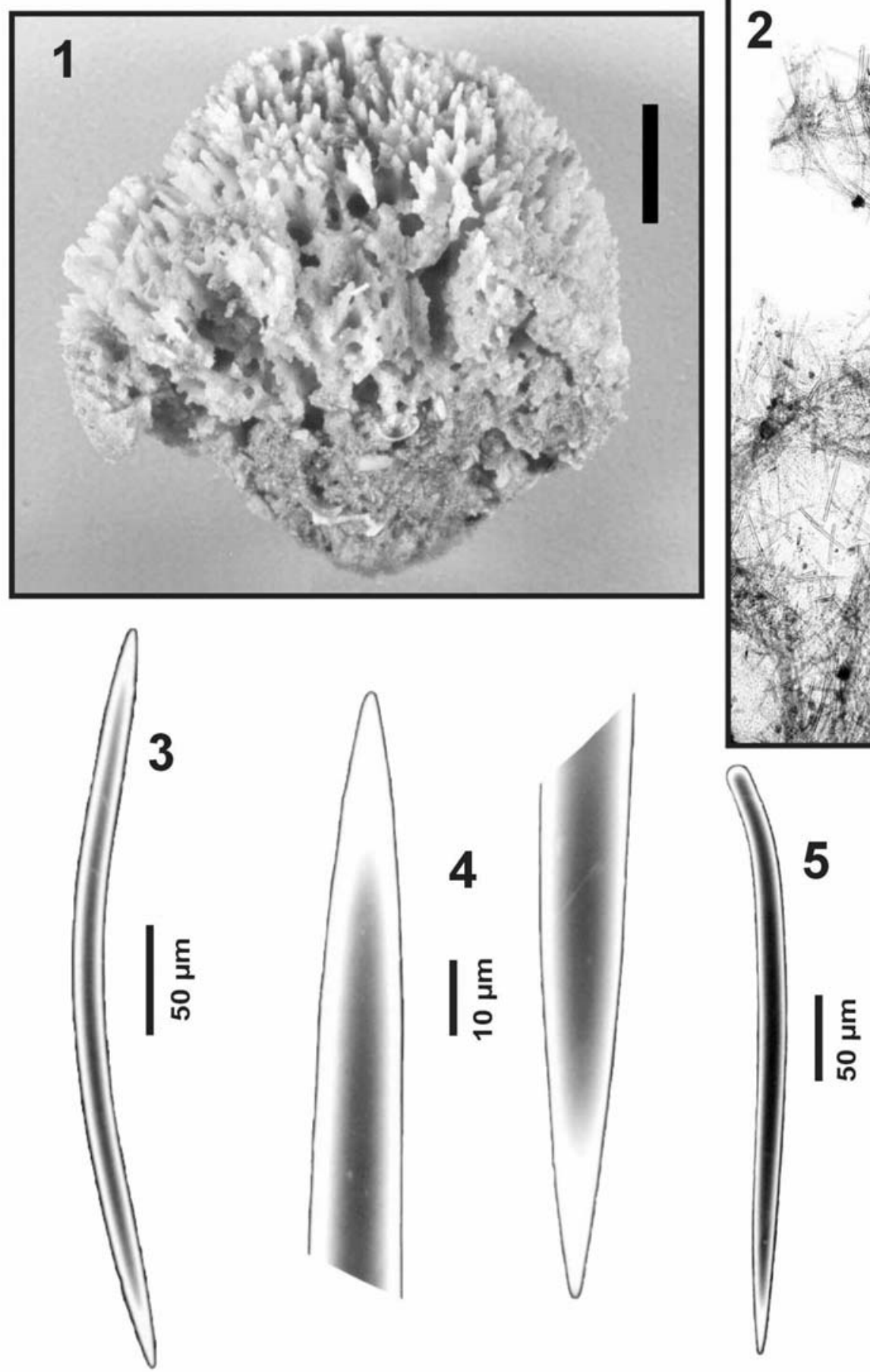

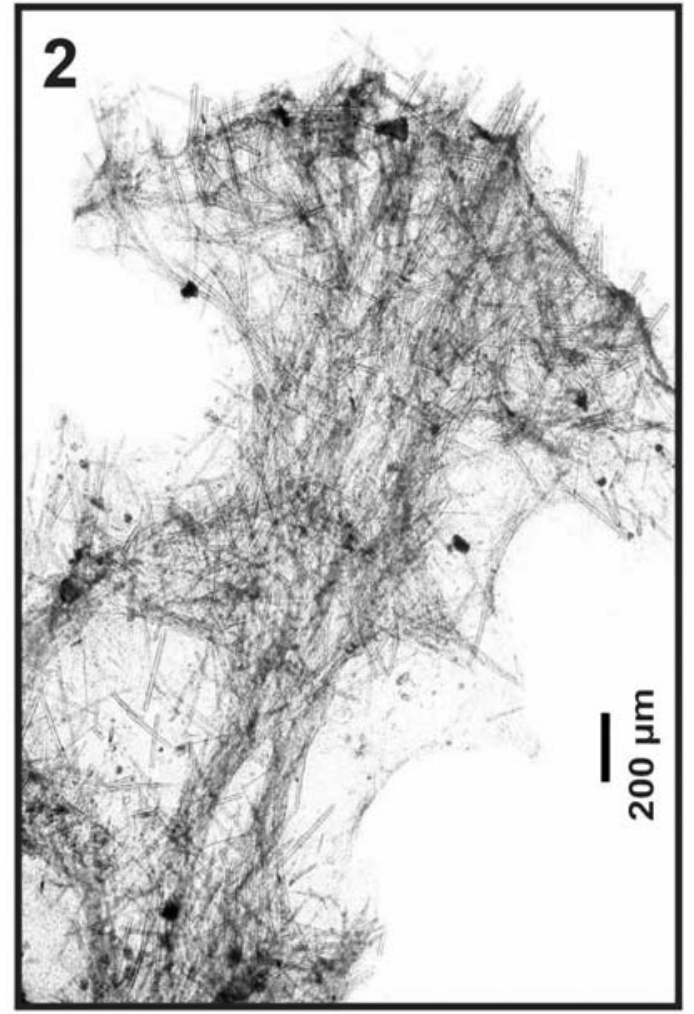

5

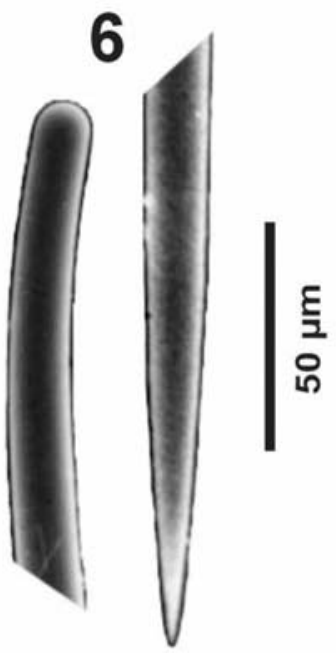

Figs. 1-6. Dragmacidon reticulatus (Ridley \& Dendy, 1886): 1, MCN 3804 (escala, $1 \mathrm{~cm}$ ); 2, arquitetura esqueletal em corte perpendicular; 3 , óxea; 4 , extremidades da óxea; 5 , estilo; 6 , extremidades do estilo 
Comentários. As dimensões das escleras dos espécimes do Caribe (Alvarez et al., 1998) e da costa brasileira se mantêm num mesmo padrão (RIDLEY \& DENDY, 1886; M.-DE-Moraes, 1987 e LERnER, 1996), inclusive as da costa do Maranhão.

Distribuição geográfica. Atlântico ocidental: Carolina do Norte, Carolina do Sul, Georgia, Florida, Bermuda, Golfo do México, Belize, Nicarágua, Porto Rico, Ilhas Virgens, Curaçao, Tobago, Venezuela (Alvarez et al., 1998); Brasil: Amapá, ao largo da desembocadura do Rio Amazonas (Collette \& RÜTZler, 1977); Maranhão (novo registro); Pernambuco (Hechtel, 1976); Atol das Rocas, Rio Grande do Norte (Moraes et al., 2003); Bahia (Ridley \& Dendy, 1886); Espírito Santo (Solé-Cava et al., 1981); Rio de Janeiro (Muricy et al., 1991); Santa Catarina (M.-DE-MoRAES, 1987; LERNER, 1996).

Distribuição batimétrica. Desde 1,5 m (Santa Catarina) até $80 \mathrm{~m}$.

Tabela I. Dragmacidon reticulatus (Ridley \& Dendy, 1886): mensurações das escleras.

\begin{tabular}{ccc}
\hline MCN & Estilos & Óxeas \\
\hline 3804 & $171-247-313,5 /$ & $218,5-281,4-342 /$ \\
& $2,3-10,5-15$ & $4,6-8,2-12,7$ \\
4775 & $210-329,4-430 /$ & $230-346,4-440 /$ \\
& $2,5-10,6-17,5$ & $2,5-10,7-17,5$ \\
\hline
\end{tabular}

\section{Desmoxyidae}

\section{Myrmekioderma rea (Laubenfels, 1934)}

(Figs. 7-14)

Anacanthea rea LAubenfels, 1934:11, 12 (localidade-tipo: Porto Rico, Caribe).

Myrmekioderma rea; Diaz et al., 1993; Lehnert \& Soest, 1998:85.

Sinonímia adicional, DiAZ et al. (1993).

Material examinado. BRASIL, Maranhão: $01^{\circ} 33$ ' 81 '’S$43^{\circ} 15^{\prime} 87^{\prime \prime} \mathrm{W}, 80$ m, 02.XII.1997 (MCN 3810, 3819, 3948).

Espécime maciço, amorfo (fig. 7). Dimensões do maior espécime (MCN 3819), em cm: comprimento 11,8, largura 6,0, altura 3,0. Superfície discretamente híspida ao tato, pronunciadamente tuberculada; superfície microverrugosa sobre a parede dos tubérculos em algumas regiões; ósculos circulares na porção superior de alguns tubérculos; ósculos 0,6-0,7 cm em diâmetro. Material preservado de consistência discretamente compressível; coloração marrom-clara.

Ectossoma (fig. 8) destacável, formado por paliçada de óxeas microespinhadas. Coanossoma com óxeas lisas abundantes, superiores paratangenciais e inferiores em discretos feixes ascendentes (80-120 $\mu \mathrm{m}$ de espessura), com pouca espongina e contendo 5-8 escleras. Raras atravessam o ectossoma, protraindo na superfície; escleras conectadas por raros feixes transversais $(30-55 \mu \mathrm{m}$ de espessura), contendo 2-3 escleras ou escleras isoladas; raras escleras microespinhadas do ectossoma, dispostas aleatoriamente, também observadas no coanossoma. Tricodragmas não observadas.

Óxeas I (figs. 9, 10) microespinhadas, espinhos concentrados nas extremidades, região mediana algumas vezes sem microespinhadura; discretamente encurvadas, raras infladas na porção mediana; extremidades gradualmente aceradas. Dimensões (tab. II).

Óxeas II (figs. 11-14) lisas, discretamente encurvadas, algumas retas, canal axial algumas vezes visível, extremidades gradualmente aceradas, raras estrongilóxeas. Dimensões (tab. II).

Comentários. Para o Brasil e Caribe ocorrem duas espécies, Myrmekioderma rea e M. gyroderma (Alcolado, 1984). Conforme Dr. Sven Zea e Dr. Pedro Alcolado (com. pess.), $M$. rea é uma espécie psamófila, vivendo enterrada entre areia e cascalho ou, quando em substrato duro, nos arrecifes, encontra-se preenchendo cavidades, sendo também morfologicamente distinta de $M$. gyroderma, a qual apresenta sulcos serpentiformes na sua superfície.

A identificação dos espécimes foi com base em comparação com o material identificado por S. Zea (INV 528). A única diferença observada é que o material da costa do Maranhão aqui estudado não apresenta tricodragmas. Como a presença deste tipo de esclera é uma característica do gênero, esta ausência é aqui considerada um fenômeno atípico. Este fenômeno de alternância de presença e ausência de uma esclera tem sido observado também com a esclera do tipo sigma, em Niphates erecta Duchassaing \& Michelotti, 1864, na região do Caribe (SoEST, 1980).

Distribuição geográfica. Atlântico ocidental: Bahamas (Diaz et al., 1993); Cuba (Alcolado \& Gotera, 1986); Porto Rico (Laubenfels, 1934); Barbados (Soest \& Stentoft, 1988); Venezuela (Diaz et al., 1993); Brasil: Maranhão (novo registro); Rio Grande do Sul (RosABARBOSA, 1995).

Distribuição batimétrica. Desde $20 \mathrm{~m}$ (Cuba) até $100 \mathrm{~m}$ (Barbados).

Tabela II. Myrmekioderma rea (Laubenfels, 1934): mensurações das escleras.

\begin{tabular}{ccc}
\hline MCN & Óxeas I & Óxeas II \\
\hline 3810 & $228-256,3-332,5 /$ & $731,5-820,8-902,5 /$ \\
& $6,9-8,1-9,2$ & $20,7-25-28,8$ \\
3819 & $228-262,2-351,5 /$ & $693,5-806,4-907,3 /$ \\
3948 & $5,8-8,2-11,5$ & $18,4-24-28,8$ \\
& $209-264,9-304 /$ & $779-887,6-978,5 /$ \\
& $4,6-7,7-9,2$ & $23-26,7-32,2$ \\
\hline & &
\end{tabular}

Topsentia ophiraphidites (Laubenfels, 1934) (Figs. 15-20)

Viles ophiraphidites Laubenfels, 1934:13, 14 (localidade-tipo: Porto Rico, Caribe).

Topsentia ophiraphidites; LeHNERT \& SOEST, 1998:85; Muricy \& Moraes, 1998:215; Lehnert \& Soest, 1999:150; Moraes et al., 2003:17.

Sinonímia adicional, DiAz et al. (1993).

Material examinado. BRASIL, Maranhão: $00^{\circ} 50^{\prime} \mathrm{N}-$ $43^{\circ} 58^{\prime} \mathrm{W}, 49 \mathrm{~m}, 15 . \mathrm{VII} .2001$ (5332, 5339); 00²2'N-445' $\mathrm{W}$,

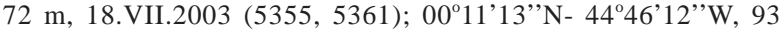
m, 10.VI.1999 (4764, 5002); 00²0'38''S-4417'38''W, 39 m, 12.VI.1999 (5015); 00³2’S-4350’W, 72 m, 18.XI.1997 (3825); 
00³5'12"'S-43'20'55'W, 94 m, 14.VI.1999 (4791, 4915, 4794, 4796, 4799, 4801, 4802, 4999); 0051'44''S-44²1'24'”W, 31 m, 12.VI.1999 (4782); 01 $01^{\circ} 25^{\prime \prime} \mathrm{S}-43^{\circ} 30^{\prime} 36^{\prime \prime} \mathrm{W}, 184 \mathrm{~m}$, 15.VI.1999 (4805); 01³3'81'"S-4315'87'”, 80 m, 02.XII.1997 (3453); 01 54 '31' 'S-44 03'43',W, 30 m, 17.XI.1997 (3847); $01^{\circ} 57^{\prime} 01^{\prime \prime} \mathrm{S}-42^{\circ} 24^{\prime} 15^{\prime \prime} \mathrm{W}, 70 \mathrm{~m}, 05 . X I I .1997$ (3801). Todos depositados no MCN.

Espécime maciço, amorfo (fig. 15). Dimensões do maior espécime (MCN 3825), em cm: comprimento 12,5, largura 8,0, altura 5,8. Superfície lisa ao tato, tuberculada; ósculos circulares, raros, distribuídos pela superfície; ósculos 0,1-1,2 cm em diâmetro. Material preservado de consistência dura; coloração amarela-clara.

Esqueleto (figs. 16,17) halicondróide, com grande concentração de óxeas desorganizadas e espongina reduzida. Ectossoma com óxeas I, de arranjo paratangencial. Coanossoma com grande quantidade de escleras do tipo óxeas II sem padrão aparente.

Oxeas I e II (figs. 18-20) lisas, discretamente curvadas na região basal, extremidades obtusas. Dimensões (tab. III).
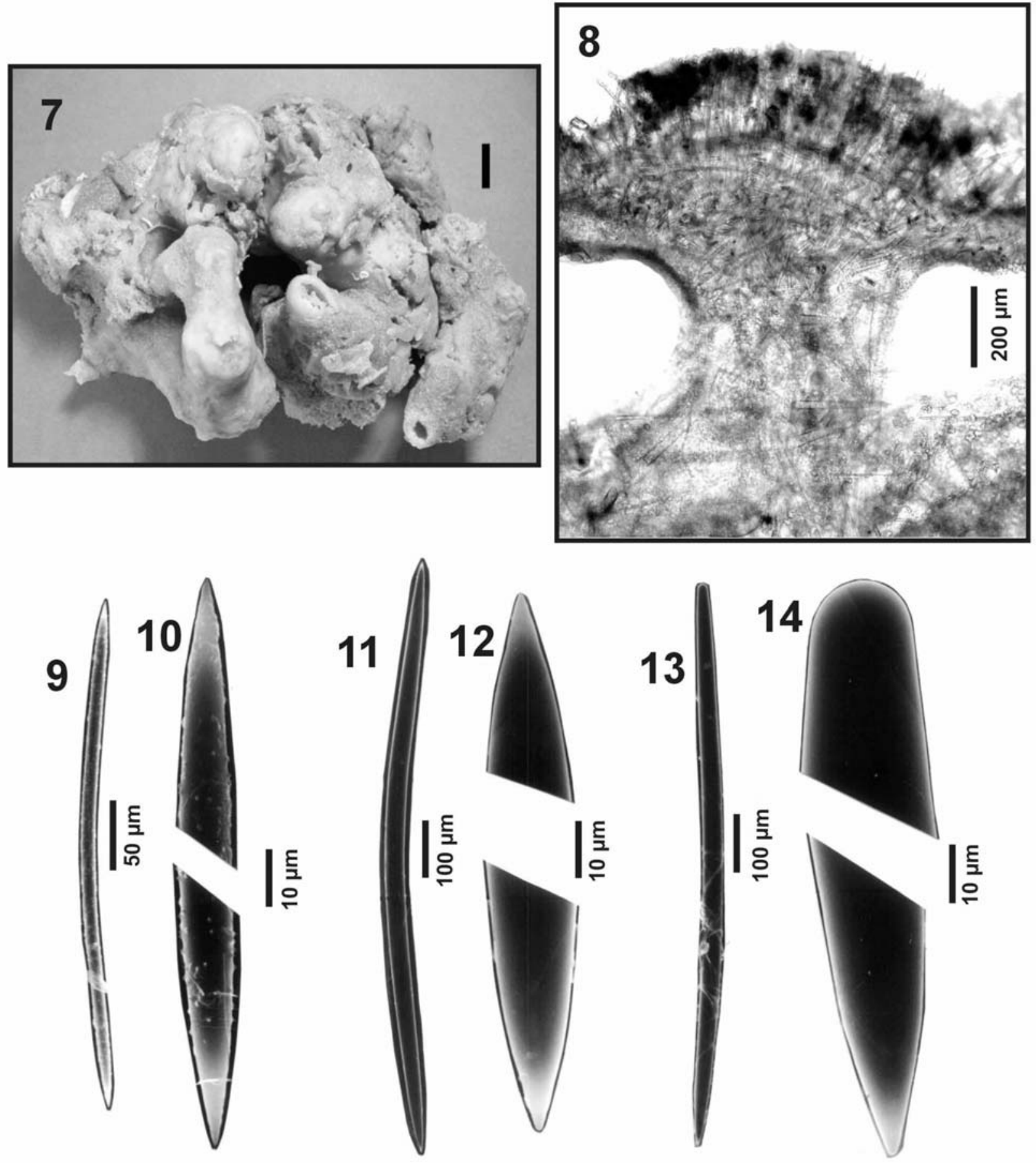

Figs. 7-14. Myrmekioderma rea (Laubenfels, 1934): 7, MCN 3819 (escala, $2 \mathrm{~cm}$ ); 8, arquitetura esqueletal em corte perpendicular; 9, óxea I; 10, extremidades da óxea I; 11, óxea II; 12, extremidades da óxea II; 13, estrongilóxea; 14, extremidades da estrongilóxea. 
Comentários. DiAz et al. (1993) referiram a ocorrência de duas a três categorias de óxeas. No material estudado, foi possível distinguir somente duas categorias, onde a menor encontra-se disposta no ectossoma. A identificação específica foi por comparação com o material identificado pelo Dr. Rob van Soest (ZMA 8805).

Distribuição geográfica. Atlântico ocidental: Caribe. República Dominicana (Diaz et al., 1993); Porto Rico
(LAubenfels, 1934); Barbados (Soest \& Stentoft, 1988); Curaçao, Colombia e Venezuela (DiAz et al., 1993); Brasil: Maranhão (novo registro); Atol das Rocas, Rio Grande do Norte (Moraes et al., 2003); Pernambuco (Hechtel, 1983); Fernando de Noronha e Tamandaré (Muricy \& MORAEs, 1998).

Distribuição batimétrica. Desde 20 m (República Dominicana) até $184 \mathrm{~m}$.
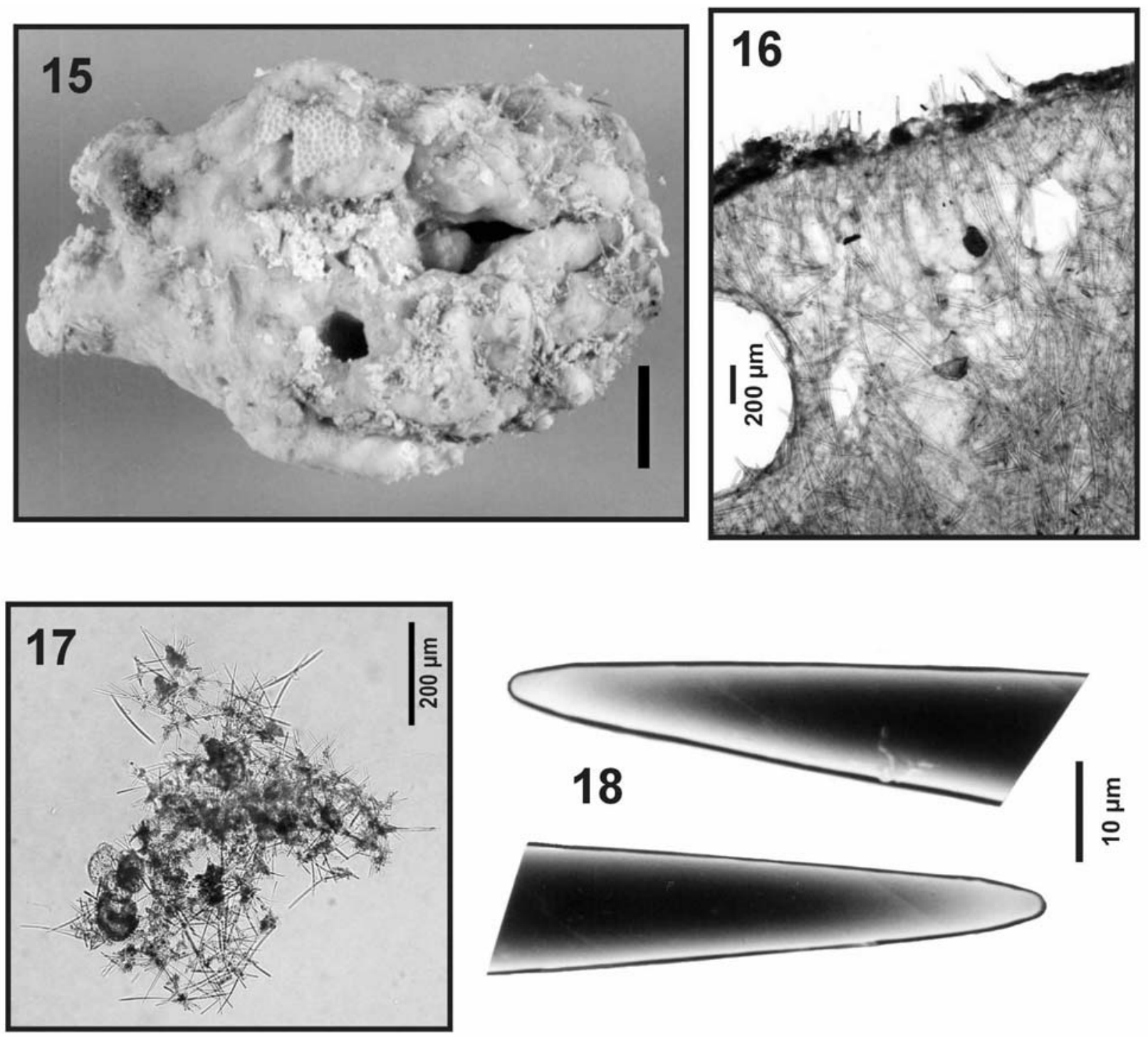

19

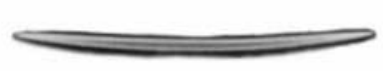

20

Figs. 15-20. Topsentia ophiraphidites (Laubenfels, 1934): 15, MCN 3825 (escala, $1 \mathrm{~cm}$ ); 16, arquitetura esqueletal em corte perpendicular; 17, arquitetura esqueletal em corte tangencial; 18, extremidades das óxeas; 19, óxea I; 20, óxea II. 
Tabela III. Topsentia ophiraphidites (Laubenfels, 1934): mensurações das escleras; $\mathrm{N}=25$.

\begin{tabular}{|c|c|c|}
\hline $\mathrm{MCN}$ & Óxeas I & Óxeas II \\
\hline 5332 & $\begin{array}{c}200-289,6-380 / \\
2,5-3,8-6,3\end{array}$ & $\begin{array}{c}390-607,8-770 / \\
6,3-12,6-18,8\end{array}$ \\
\hline 5339 & $\begin{array}{c}210-285,2-360 / \\
2,5-4,1-6,3\end{array}$ & $\begin{array}{c}380-598,4-770 / \\
6,3-11,4-17,5\end{array}$ \\
\hline 5355 & $\begin{array}{c}210-303,5-370 / \\
2,5-5-7,5\end{array}$ & $\begin{array}{c}390-651,6-950 / \\
7,5-12,6-18,8\end{array}$ \\
\hline 5361 & $\begin{array}{c}190-269,2-380 / \\
2,5-3,8-6,3\end{array}$ & $\begin{array}{c}400-624,8-820 / \\
6,3-13,7-20\end{array}$ \\
\hline 5002 & $\begin{array}{c}110-143,3-170 / \\
2,5-3,4-5\end{array}$ & $\begin{array}{c}410-613,2-800 / \\
7,5-13,9-21,3\end{array}$ \\
\hline 4764 & $\begin{array}{c}160-266,8-340 / \\
2,5-5,3-7,5\end{array}$ & $\begin{array}{c}370-648,4-950 / \\
7,5-17,4-28,8\end{array}$ \\
\hline 5015 & $\begin{array}{c}170-254,6-360 / \\
2,5-5,3-7,5\end{array}$ & $\begin{array}{c}400-656,7-890 / \\
7,5-16-25\end{array}$ \\
\hline 3825 & $\begin{array}{c}228-295,6-380 / \\
2,3-5,4-8,1\end{array}$ & $\begin{array}{c}400-681-950 / \\
6,9-15-23\end{array}$ \\
\hline 4791 & $\begin{array}{c}210-290-370 / \\
3,8-6,7-8,8\end{array}$ & $\begin{array}{c}380-641,6-930 / \\
8,8-16,6-27,5\end{array}$ \\
\hline 4915 & $\begin{array}{c}160-266,5-360 / \\
3,8-5,6-8,8\end{array}$ & $\begin{array}{c}400-671,4-950 / \\
7,5-16,9-27,5\end{array}$ \\
\hline 4794 & $\begin{array}{c}170-290,4-370 / \\
2,5-4,1-6,3\end{array}$ & $\begin{array}{c}440-684,2-990 / \\
7,5-15,5-25\end{array}$ \\
\hline 4796 & $\begin{array}{c}220-320,4-390 / \\
2,5-4,1-6,3\end{array}$ & $\begin{array}{c}480-800-1130 / \\
6,3-14,9-27,5\end{array}$ \\
\hline 4799 & $\begin{array}{c}210-294,2-380 / \\
3,8-5,5-8,8\end{array}$ & $\begin{array}{c}390-663,8-1010 \\
7,5-15,9-27,5\end{array}$ \\
\hline 4801 & $\begin{array}{c}209-304,4-380 / \\
2,5-3,9-6,3\end{array}$ & $\begin{array}{c}410-676,8-960 / \\
5-14,2-22,5\end{array}$ \\
\hline 4802 & $\begin{array}{c}170-282-380 / \\
2,5-4,7-7,5\end{array}$ & $\begin{array}{c}430-753,2-1300 / \\
7,5-16-28,8\end{array}$ \\
\hline 4999 & $\begin{array}{c}160-285,2-370 / \\
2,5-3,9-6,3\end{array}$ & $\begin{array}{c}380-653,8-900 / \\
5-12,7-20\end{array}$ \\
\hline 4782 & $\begin{array}{c}190-301,9-380 / \\
2,5-3,9-6,3\end{array}$ & $\begin{array}{c}430-683,2-1010 / \\
7,5-12-18,8\end{array}$ \\
\hline 4805 & $\begin{array}{c}170-299,2-390 / \\
3,8-6-8,8\end{array}$ & $\begin{array}{c}450-743,8-1450 \\
7,5-20,9-40\end{array}$ \\
\hline 3453 & $\begin{array}{c}230-288-370 / \\
3,8-6,9-10\end{array}$ & $\begin{array}{r}390-661-970 / \\
8,8-18,2-27,5\end{array}$ \\
\hline 3847 & $\begin{array}{c}160-279,2-390 / \\
2,5-3,8-6,3\end{array}$ & $\begin{array}{c}390-654,4-910 / \\
6,3-14,1-22,5\end{array}$ \\
\hline 3801 & $\begin{array}{c}180-282,8-380 / \\
2,5-5,6-8,8\end{array}$ & $\begin{array}{c}350-622,6-900 / \\
7,5-17,1-27,5\end{array}$ \\
\hline
\end{tabular}

Agradecimentos. Ao Dr. Sven Zea (INV) e Dr. Pedro Alcolado, Instituto de Oceanologia, Academia de Ciências de Cuba, pela doação de material para estudo comparativo e pelos valiosos comentários. Ao Dr. Rob van Soest (ZMA), pelo empréstimo de material para estudo comparativo.

\section{REFERÊNCIAS BIBLIOGRÁFICAS}

Alcolado, P. M. \& Gotera, G. G. 1986. Nuevas adiciones a la fauna de poríferos de Cuba. Poeyana, La Habana, 331:1-19.

Alvarez, B. \& Hooper, J. N. A. 2002. Family Axinellidae Carter, 1875. In: Hooper, J. N. A. \& Soest, R. W. M. van eds. Systema Porifera: a guide to the classification of sponges. New York, Kluwer Academic. v.1, p.724-747.

Alvarez, B.; Soest, R. W. M. van \& Rützler, K. 1998. A revision of Axinellidae (Porifera: Demospongiae) of the Central West Atlantic region. Smithsonian Contributions to Zoology, Washington, 598:1-47.

Coelho, E. P. \& Mello-Leitão, A. 1978. Placospongia carinata e sua ocorrência em costas brasileiras. Centro de estudos zoológicos, Universidade do Brasil, Rio de Janeiro, 29:1-12.

Collette, B. \& Rützler, K. 1977. Reef fishes over sponge bottoms off the mouth of the Amazon river. Proceedings of Third International Coral Reef Symposium, Miami, p.305-310. Diaz, M. C.; Pomponi, S. A. \& Soest, R. W. M. van. 1993. A systematic revision of the Central-Atlantic Halichondrida (Demospongiae, Porifera). Part III: description of valid species. Scientia Marina, Barcelona, 57(4):283-306.

Hajdu, E. \& Desqueyroux-Faúndez, R. 1994. A synopsis of South American Mycale (Mycale) (Poecilosclerida, Demospongiae), with the description of three new species and a cladistic analysis of Mycalidae. Revue Suisse de Zoologie, Genève, 101 (3):563600 .

Hechtel, G. J. 1976. Zoogeography of Brazilian marine Demospongiae. In: Harrison, F. W. \& Cowden, R. R. eds. Aspects of sponge biology. New York, Academic. p.237-260.

1983. New species of marine Demospongiae from Brazil. Iheringia, Série Zoologia, Porto Alegre, (63):59-89.

Laubenfels, M. W. DE. 1934. New sponges from the Puerto Rican deep. Smithsonian Miscellaneous Collections, Washington, 91(17): 1-28.

Lehnert, H. \& Soest, R. W. M. van. 1998. Shallow water sponges of Jamaica. Beaufortia, Amsterdam, 48(5):71-103.

_. 1999. More north Jamaica deep fore-reef sponges. Beaufortia, Amsterdam, 49(12):141-169.

Lerner, C. B. 1996. Esponjas da Ilha da Galé (Porifera, Demospongiae), Reserva Biológica Marinha do Arvoredo, Santa Catarina, Brasil. Biociências, Porto Alegre, 4(2):101-124.

Moraes, F. C.; Vilanova, E. P. \& Muricy, G. 2003. Distribuição das esponjas (Porifera) na Reserva Biológica do Atol das Rocas, Nordeste do Brasil. Arquivos do Museu Nacional, Rio de Janeiro, 61(1):13-22.

Mothes-DE-Moraes, B. 1978. Esponjas tetraxonidas do litoral sulbrasileiro: II. Material coletado pelo N/Oc. "Prof. W. Besnard" durante o Programa RS. Boletim do Instituto de Oceanografia, São Paulo, 27(2):57-78.

1987. Ocorrência de poríferos na zona de maré da Ilha de João da Cunha, Porto Belo, Santa Catarina, Brasil (Porifera, Demospongiae). Iheringia, Sér. Zoologia, Porto Alegre, (66):129-139.

Mothes, B.; Hajdu, E. \& Soest, R. W. M. van. 2000. Tedania brasiliensis new species (Demospongiae, Poecilosclerida, Tedaniidae) from Brazil, with some remarks about the genus Tedania on the tropical southwesthern Atlantic. Bulletin of Marine Science, Miami, 66(1):1-11.

Mothes, B.; Lerner, C. B. \& Silva, C. M. M. 1999. Revision of Brazilian Erylus (Porifera, Demospongiae) with description of a new species. Memoirs of the Queensland Museum, Brisbane, 44:369-380.

Muricy, G.; Hajdu, E. et al. 1991. Sponge distribution at Arraial do Cabo, SE Brazil. In: Symposium on Coastal Ocean Management, $7^{\text {th }}$, 1991, Long Beach. Proceedings..., ASCE Publications. p.11831196.

Muricy, G. \& Moraes, F. C. 1998. Marine sponges of Pernambuco State, NE Brazil. Revista Brasileira de Oceanografia, São Paulo, 46(2):213-217.

Ridley, S. O. \& Dendy, A. 1886. Preliminary report on the Monaxonida collected by H. M. S. "Challenger". Parts I and II Annals and Magazine of Natural History, London, (5) 18:325-351/470-493.

Rosa-Barbosa, R. DE. 1995. Primeiro registro de Myrmekioderma styx Laubenfels, 1953 (Porifera-Demospongiae) no Atlântico sudoeste com novos aportes para a caracterização da espécie. Biociências, Porto Alegre, 3(2):119-128.

Silva, C. M. M. \& Mothes, B. 1996. SEM analysis: an important instrument in the study of marine sponges biodiversity. Acta Microscopica, Rio de Janeiro, 5(B):188-189.

Soest, R. W. M. van. 1980. Marine sponges of Curaçao and other caribbean localities. Part II. Haplosclerida. Studies on the fauna of Curaçao and other Caribbean Islands, Amsterdam, 62(191): 1-173.

Soest, R. W. M. van \& Stentoft, N. 1988. Barbados deep water sponges. Studies on the fauna of Curaçao and other Caribbean Islands, Amsterdam, 70:1-175.

Solé-Cava, A. M.; Kelecom, A. \& Kannengiesser, G. J. 1981. Study of some sponges (Porifera, Demospongiae) from the infralitoral of Guarapari, Espírito Santo, Brazil. Iheringia, Sér. Zoologia, Porto Alegre, (60):125-150.

Recebido em agosto de 2003. Aceito em março de 2004. ISSN 0073-4721 